\title{
Customizable Cryo-EM Chips Improve 3D Analysis of Macromolecules
}

\author{
A. Cameron Varano ${ }^{1,2,3,4,5^{*}}$, Nick Alden ${ }^{1}$, William Dearnaley ${ }^{1,3,4,5}$, Michael Casasanta ${ }^{3,4,5}$, John \\ Damiano $^{6}$, Jennifer McConnell ${ }^{6}$, Madeline Dukes ${ }^{6}$ and Deborah F. Kelly ${ }^{1,3,4,5}$ \\ 1. Virginia Tech Carilion Research Institute, Virginia Tech, Roanoke, VA, USA. \\ 2. Translational Biology, Medicine and Health, Virginia Tech, Blacksburg, VA, USA. \\ 3. Department of Biomedical Engineering, Pennsylvania State University, University Park, PA, USA. \\ 4. Huck Institutes of the Life Sciences, Pennsylvania State University, University Park, PA, USA. \\ 5. Center for Structural Onocology, Pennsylvania State University, University Park, PA, USA. \\ 6. Application Science, Protochips Inc., Morrisville, NC, USA. \\ * Correspondence to: cvarano@psu.edu
}

The field of cryo-EM has seen immense changes in the past decade. These efforts have focused on automating data collection procedures and improving computational routines [1]. However, changes in sample preparation have remained largely unchanged. Typical sample preparation begins with a specimen applied to carbon-based support films containing holes. Then after a gentle blotting procedure, biological macromolecules are rapidly frozen. Alternative materials, such as gold foils and graphene, have begun to replace traditional materials in the cryo-EM field, and have proven useful for sample preparation procedures [2]. However, these substrates remain limited in their ability to mediate ice thickness and protect fragile macromolecules. We aim to develop new tools to help streamline studies for the scientific community.

In order to more precisely manage ice thickness and sample integrity, we radically redesigned the substrate- utilizing wells rather than holes to capture specimen (Figure 1). The new custom-designed Silicon Nitride $(\mathrm{SiN})$ microchips $\left(\right.$ Cryo-Chip $\left.{ }^{\mathrm{TM}}\right)$ contain integrated windows. The window dimensions range from 5-10 $\mu \mathrm{m}$ in $\mathrm{x}$ - and $\mathrm{y}$-dimensions. Previous microchips contained larger imaging windows (200 - $500 \mu \mathrm{m}$ ), which while sufficient for cryo-EM [3] were more susceptible to break during freezing and loading procedures. The newly-engineered topographies provide three key advantages. First, the integrated micro-wells provide additional stability during freezing. Secondly, they provide a physical mediator for more consistent ice thickness. Finally, they enhance particle retention and integrity on the substrate. The micro-well structures appear to mitigate the harmful effects of the air-water interface. It is the air-water interface where the aggregation and denaturation commonly occur and interfere with structural studies [2].

To evaluate the Cryo-Chip design we examined two very different macromolecules, rotavirus and BRCA1-assemblies, on the FEI Twin BioSpirit TEM under low-dose conditions $\left(<10\right.$ electrons $\left./ \AA^{2}\right)$ at 120 $\mathrm{kV}$. Rotavirus is a larger complex ( $\sim 80 \mathrm{~nm}$ diameter) with icosahedral symmetry. BRCA1- associated assemblies are smaller $(\sim 17 \mathrm{~nm}$ across $)$, highly flexible and non-symmetric. We were able to more consistently replicate the freezing conditions on the Cryo-Chips as compared to the traditional substrate. Computationally, we observed enhanced image contrast in both biological specimens compared to conventional holey carbon films (Figure 2). Higher contrast allowed for more accurate computing procedures. Here we demonstrate that Cryo-Chips are a powerful tool for examining a variety of biological samples [4]. 
References:

[1] S. H. Scheres, Journal of Structural Biology 180 (2012), p. 519.

[2] R. M. Glaeser, Nature Methods 13 (2016), p. 28.

[3] C. E. Winton et al., Microscopy Today 25(4) (2017). p. 22.

[4] The authors acknowledge the following funding sources that supported this work NIH/NCI R01CA193578, R01CA227261, R01CA219700.

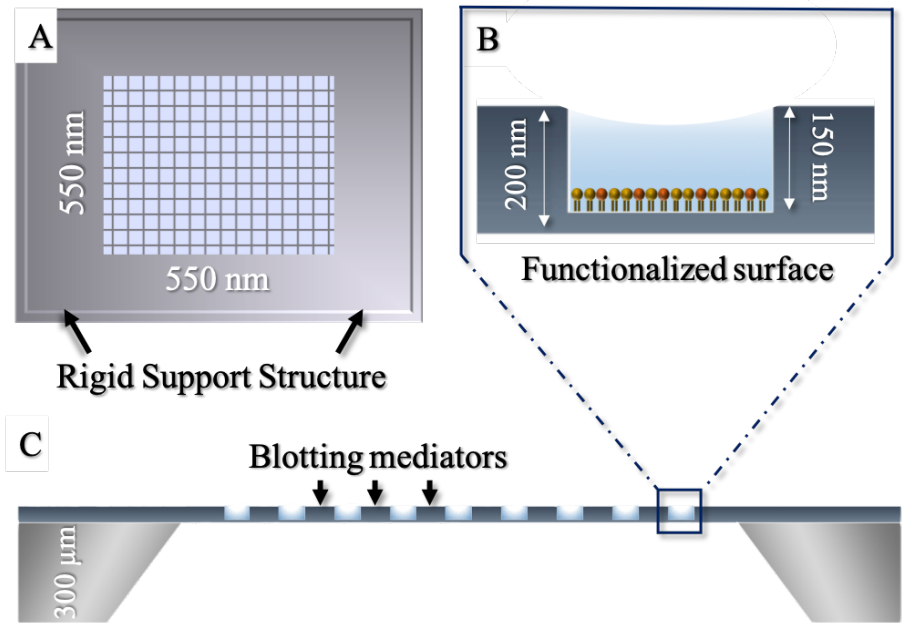

Figure 1. Cryo-Chips with integrated micro-wells mediated freezing process. (a) View of Silicon Nitride microchips with integrated wells. (b) Side view of a single functionalized micro-well. (c) Side view of microchip shows micro-wells provide a physical mediator for blotting.
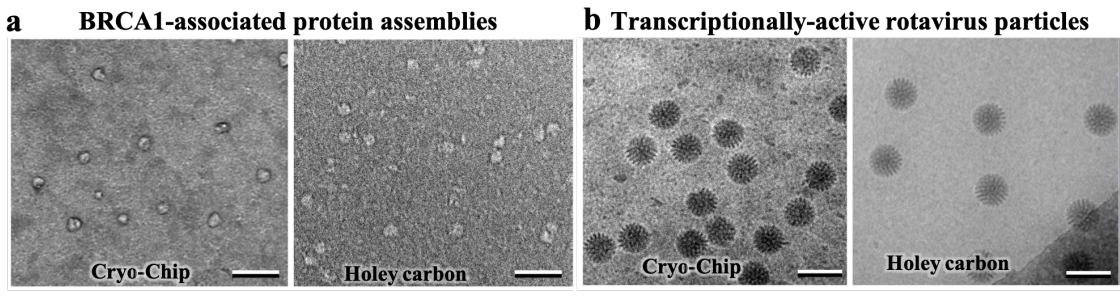

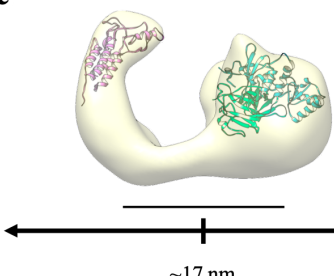

BRCA1 assemblies contain a flexible central region

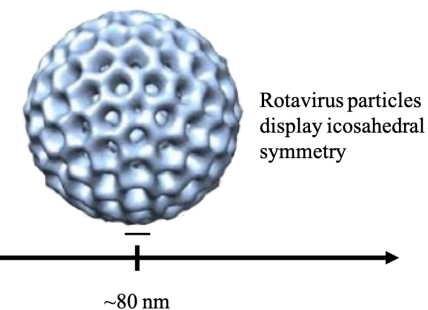

Figure 2. Cryo-Chip provides is a higher contrast substrate for biological samples. (a) EM image of BRCA1-associated assemblies imaged on Cryo-Chip have higher contrast compared to traditional holey carbon. Scale bar is $100 \mathrm{~nm}$. (b) Rotavirus particles imaged on Cryo-Chip have higher contrast compared to traditional holey carbon. Scale bar is $100 \mathrm{~nm}$. (c) BRCA1 assemblies are smaller, non-symmetrical and contain a highly flexible central region while (d) rotavirus particles are larger with icosahedral symmetry. Scale bars are $10 \mathrm{~nm}$. 